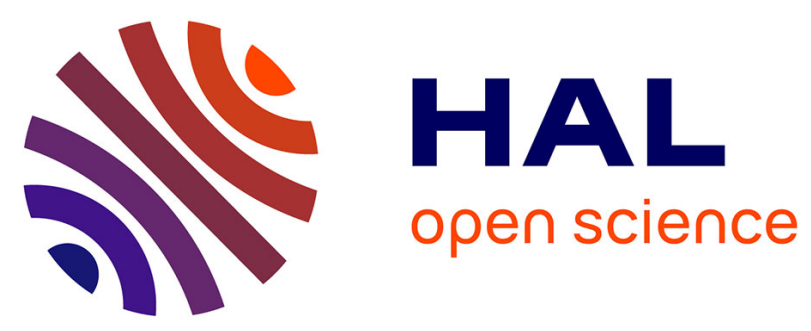

\title{
Deep Group-Wise Angular Translation of Cardiac Diffusion MRI in q-space via Manifold Regularized GAN
}

Yunlong He, Lihui Wang, Feng Yang, Patrick Clarysse, Yue-Min Zhu

\section{To cite this version:}

Yunlong He, Lihui Wang, Feng Yang, Patrick Clarysse, Yue-Min Zhu. Deep Group-Wise Angular Translation of Cardiac Diffusion MRI in q-space via Manifold Regularized GAN. 2020 15th IEEE International Conference on Signal Processing (ICSP), Dec 2020, Beijing, China. pp.511-515, 10.1109/ICSP48669.2020.9320925 . hal-03435215

\section{HAL Id: hal-03435215 https://hal.science/hal-03435215}

Submitted on 18 Nov 2021

HAL is a multi-disciplinary open access archive for the deposit and dissemination of scientific research documents, whether they are published or not. The documents may come from teaching and research institutions in France or abroad, or from public or private research centers.
L'archive ouverte pluridisciplinaire HAL, est destinée au dépôt et à la diffusion de documents scientifiques de niveau recherche, publiés ou non, émanant des établissements d'enseignement et de recherche français ou étrangers, des laboratoires publics ou privés. 


\title{
Deep Group-Wise Angular Translation of Cardiac Diffusion MRI in q-space via Manifold Regularized GAN
}

\author{
Yunlong He, Lihui Wang*, Feng Yang, Patrick Clarysse, and Yuemin Zhu*
}

\begin{abstract}
Diffusion magnetic resonance imaging (dMRI) has become an indispensable tool for non-invasive characterization of fiber structures of tissues. Clinical applicability of dMRI is often shackled by trade-off between image quality and long acquisition time. We propose a novel group-wise image translation method to improve the angular resolution of cardiac dMRI data. It consists in using a generative adversarial network (GAN) model to estimate a sequence of images from given DW images acquired in a limited number of diffusion gradient directions. We embed a supervised manifold regularized term in the GAN loss function to exploit the correlation between multiple DW images acquired in different gradient directions. Experimental results on cardiac dMRI data demonstrated that our method can significantly improve the quality of diffusion tensor imaging (DTI) reconstruction.
\end{abstract}

Index Terms-diffusion MRI, super-resolution, cardiac DTI, image synthesis, deep learning, spatial-angular information

\section{INTRODUCTION}

Diffusion magnetic resonance imaging (dMRI) is a noninvasive imaging modality that probes the displacement of the water molecules using diffusion-weighted pulse sequences. It has been widely used to study diffusion properties and fiber structures of biological tissues at a microscopic scale. In cardiac imaging, dMRI provides an avenue to characterize myocardial fiber architecture and has been used in various applications and diseases [1].

Signal reconstruction in dMRI usually involves fitting a limited number of samples of the diffusion signal in 3D q-space via an elegant model such as Diffusion Tensor Imaging (DTI) [2]. The latter acquires signals from six gradient directions and is adequate to recover the orientation of a single fiber at each voxel. However, more complex configurations (such as fiber crossing and bending) typically require more sophisticated models, e.g., High Angular Resolution Diffusion Imaging (HARDI) [3] acquires diffusion-weighted (DW) images along a large number of diffusion gradient directions, each of which corresponds to a point in q-space. A large number of DW

Y. He, P. Clarysse, and Y. Zhu are with Univ Lyon, INSA-Lyon, Université Claude Bernard Lyon 1, UJM-Saint Etienne, CNRS, Inserm, CREATIS UMR 5220, U1206, F-69621, LYON, France. (e-mail: Yunlong.He@creatis.insa-lyon.fr; patrick.clarysse@creatis.insa-lyon.fr; YueMin.Zhu@creatis.insa-lyon.fr)

L. Wang is with Key Laboratory of Intelligent Medical Image Analysis and Precise Diagnosis of Guizhou Province, School of Computer Science and Technology, Guizhou University, Guiyang, China. (E-mail: wlh1984@gmail.com).

F. Yang is with School of Computer and Information Technology, Beijing Jiaotong University, Beijing 100044, China. (E-mail: fengyang@bjtu.edu.cn).

*Corresponding author: Lihui Wang and Yuemin Zhu images increase the accuracy of DTI reconstruction, but also lengthen acquisition time, making dMRI impractical for clinical routine use.

To address such problem, many post-acquisition methods were proposed to increase the angular resolution of DW images acquired in a limited number of gradient directions. For example, [4] interpolated signals by weighted averaging of angular neighboring measurements in q-space. [5] proposed to employ spherical harmonic coefficient vectors for the measurement interpolation. While these methods achieve promising results, they neglected the fact that the signal is smooth in the joint $\mathrm{x}-\mathrm{q}$ space. To fill this gap, several compressive sensing methods were developed, in which joint $x-q$ space information from dMRI data was exploited to generate HARDI signals with a higher quality [6]-[8]. However, they usually need dedicated imaging protocols, which limits their clinical applicability.

Another possible solution to this problem is to estimate one modality image from another using learning-based methods. In this field, Jog et al. [9] generated high resolution MR images from low resolution scans by learning a nonlinear regression with random forest. Besides, the random forest was also used to synthesize CT from MRI [10]. Recent deep learning methods are attracting more and more research interests, mainly due to their ability to map real distribution of data more rapidly and more objectively without the need of manual work. For instance, Dong et al. [11] addressed the single image super-resolution using Convolutional Neural Networks (CNNs) and gained impressive results. Kim et al. [12] improved the performance of the super-resolution scheme using a recursive $\mathrm{CNN}$ with a simple parametric learning model.

Despite achieving promising performance in generating real images, existing learning-based methods were mainly designed for single medical image transformation, while it is nontrivial to apply them to DMRI image estimation due to the spatialangular information within multiple images rather than independent spatial information in a image. CNNs model may handle this problem by operating deep convolution on both spatial and angular domains using multi-channel 2D or 3D kernels. Unfortunately, most deep learning methods focused on standard medical images, such as computerized tomography (CT), positron emission tomography (PET)) or the translation of single image modality. Moreover, traditional CNN tends to minimize the simple Euclidean distance between predicted 
and ground truth pixels, which is insufficient to measure the correlation between images acquired from different gradient directions in dMRI.

In this paper, we proposed a deep leaning-based method to improve the angular resolution of cardiac dMRI data without the need of requiring a large number of dMRI scans. Different from previous works which focused on single image translation, our method is a group-wise translating strategy which consists of training a generative model to simultaneously estimate a sequence of new DW images from a sequence of given DW images acquired in a limited number of gradient directions. Instead of directly using traditional CNNs that only minimize Euclidean distance between predicted and ground truth images, we trained a generative adversarial network (GAN) [13], [14] to minimize the difference at a higher level, i.e., trying to classify whether the output image is real or fake. Moreover, we modified the traditional GAN loss function using a supervised manifold regularized term to exploit the correlation between DW images in different gradient directions.

\section{MethoD}

The goal of our method is to train a deep generative network which can predict highly angular resolved images from images acquired in a limited number of gradient directions. Given a set of DW images $X:\left\{x_{1}, x_{2}, \cdots, x_{n}\right\}$ which are acquired in $n$ diffusion gradient directions, our objective is to learn a mapping $G$ from existing $X$ to DW images in new directions $G(X):\left\{x_{n+1}, x_{n+2}, \cdots, x_{2 n}\right\}$, where $\left\{x_{1}, x_{2}, \cdots, x_{2 n}\right\}$ denotes DW images with high angular resolution and their diffusion gradient direction should be distributed uniformly on a sphere.

\section{A. GAN architecture}

We propose to use conditional GANs [15] due to their ability to generate highly realistic images with a limited number of observed DW images $X$. More precisely, we trained two networks to compete with each other, a Generator $G$ and a Discriminator $D$. Fig. 1 illustrates an overview of proposed GAN network, the Generator $G$ applies the transform to $n$ observed DW images $X$ to predict the DW images $G(X)$ in a set of new $n$ directions. The discriminator takes both the observed DW images $\mathrm{X}$ in the $\mathrm{n}$ directions and images in the $\mathrm{n}$ new directions, where the images in the new directions can be either the output images from the generator or the real images from the original dataset; and then the discriminator tries to decide if the images in new directions were produced by the generator or not. In Fig. 1 the red dashed arrows denote the back propagation of the network that adjust the network parameters to update the generator. This process continues iteratively until the generator can produce highly realistic DW image sequences.

Our network is constructed based on convolutionBatchNorm-ReLu [16] and U-Net [17] as encoder-decoder that combines the features from shallow and deep layers through multi-path information to optimize the generator quickly.

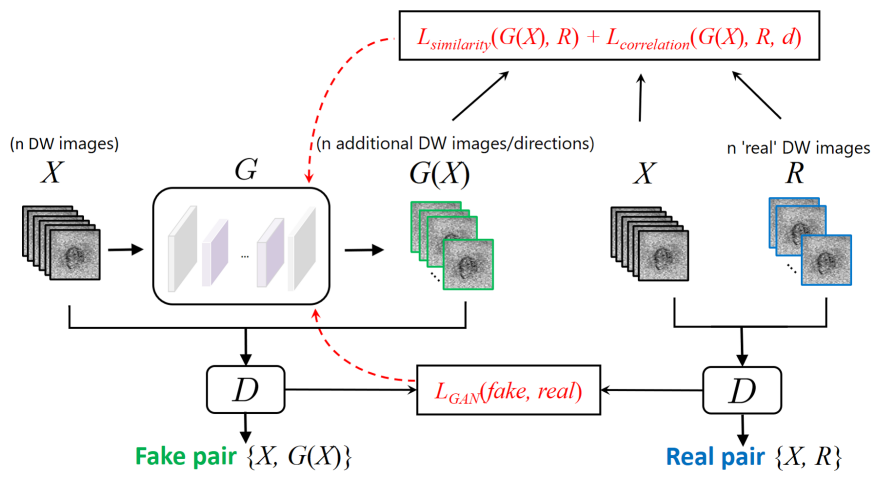

Fig. 1. Schematic illustration for the proposed manifold regularized GAN. The network maps DW images acquired in $n$ gradient directions into new $n$ directions.

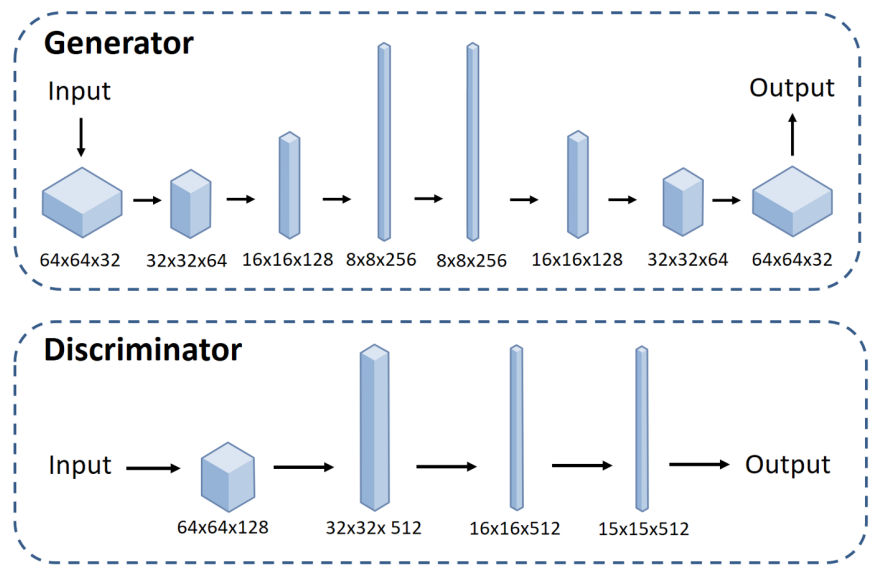

Fig. 2. The detailed network architecture of our generator and discriminator. The solid arrows indicate the forward propagation of the network. The number below the box indicates the size of the feature map in its corresponding layer.

Fig. 2 presents our network architecture in detail. For the generator, the input is $n 128 \times 128 \mathrm{DW}$ images with $n$ channels (from $n$ gradient directions), and the output is the same. The discriminator employs a PatchGAN strategy proposed in [18]. The input of discriminator is $n 128 \times 128$ DW image with $n$ channels (from $n$ gradient directions), and the output is a $15 \times 15$ image map where each pixel value represents how believable the corresponding patch of the input image is. The How number of diffusion gradient directions $n$ used in our experiments were 6,12 , and 24 .

\section{B. GAN Objective}

The loss function of conditional GAN network can be expressed as [15]:

$$
\begin{aligned}
L_{G A N}(G, D) & =E_{X, R}[\log D(X, R)] \\
& +E_{X}[\log (1-D(X, G(X)))]
\end{aligned}
$$

where $R:\left\{r_{n+1}, r_{n+2}, \cdots, r_{2 n}\right\}$ represents the ground-truth of $n$ DW images from real dataset compared to the generated images $G(X):\left\{x_{n+1}, x_{n+2}, \cdots, x_{2 n}\right\} . D$ tries to maximize the probability of assigning the correct label to both real $R$ 
and generated $\left\{x_{1}, x_{2}, \cdots, x_{2 n}\right\}$ groups. Simultaneously, $G$ tries to minimize the second term against $D$. By minimizing this function, the capability of generator $G$ and discriminator $D$ will gradually increase from the competition process until $D(X, R)$ and $D(X, G(X))$ have the same distributions. Previous studies have found that it is beneficial to combine the traditional GAN loss with L1 or L2 distance [19]. Therefore, in our method, the task of the generator is not only to produce realistic images but also to output difference between real and fake DW images in L2 sense. This L2 loss function can be expressed as:

$$
L_{\text {similarity }}=E_{X, R}\|(R-G(X))\|^{2}
$$

where $\|\cdot\|^{2}$ can be some distance function, such as Frobenius norm.

\section{Manifold regularized loss function}

GAN provide an ingenious competition scheme with the ability of to guide the generative model to produce data very close to the distribution of real data. However, our experiments showed that the traditional GAN even with L2 loss does not perform well in practice, as it neglects the continuous spatialangular information in DW image sequences. Therefore, we propose here a manifold regularized strategy to exploit the angular correlation between images in different gradient directions. This consists in additionally minimizing the following regularization function used in [20] for generator updates:

$$
\left.L_{\text {correlation }}=E_{X, R} \frac{1}{4 n^{2}} \sum_{i=1, j=1}^{2 n} \| f(G(X))-f(R)\right) \|^{2} W_{i, j} \text {. }
$$

where $f(\cdot)$ is an embedding function to extract the useful information from the raw data, $\| f(G(X))-f(R)) \|^{2}$ measures the difference between the real and generated data. If $f(x)=x$, then minimizing this term is equivalent to minimize Eq. (3). In our cases, both the generated and real data are a sequence of DW images for a number of directions. Then this term can be expressed as:

$$
\left\|G\left(x_{i}\right)-r_{i}-G\left(x_{j}\right)+r_{j}\right\|^{2} .
$$

$W_{i j}$ in Eq. (3) is a weight measures the angular similarity between $i$ th and $j$ th directions of DW images. It can be set according to the Locality Preserving Projections strategy [21] as follows:

$$
W_{i j}=e^{-\frac{\left\|d_{i, j}\right\|^{2}}{\varphi}}
$$

where $d_{i, j}$ denotes the angular difference between $i$ th and $j$ th diffusion gradient directions. $\varphi$ controls the scale of In the $\mathrm{dMRI}$, the diffusion gradient directions are often represented by vectors in $3 \mathrm{D}$ q-space, e.g., $\vec{v}_{i}$ and $\vec{v}_{j}$ corresponding to direction $i$ and $j$. Here we compute the angular difference $d_{i, j}$ by using the angle of two vectors, e.g., $d_{i, j}=\left(\vec{v}_{i} \cdot \vec{v}_{i}\right) /\left(\left|\vec{v}_{i}\right|\right.$. $\left.\left|\vec{v}_{i}\right|\right)$.

It should be noted that this choice of Eq. 4 with $W_{i j}$ incurs a heavy constraint to control the generated results. Here, if the data samples $r_{i}$ and $r_{j}$ are from different submanifolds in 3D q-space (in terms of angle location), it encourages the generated $G\left(x_{i}\right)$ and $G\left(x_{j}\right)$ to lie in different manifolds. Finally, our loss function is:

$$
G=\arg \min _{G} \max _{D} L_{G A N}+\lambda L_{\text {similarity }}+\phi L_{\text {correlation }}
$$

Where $\lambda$ and $\phi$ are regularization parameters which control the importance of the spatial similarity and angular correlation, respectively.

\section{EXPERIMENTS}

\section{A. Datasets}

dMRI of three ex vivo human hearts were acquired in clinical conditions with Siemens 3T MRI Magnetom Verio. The imaging parameters are the following: $T E=74 \mathrm{~ms}, T R=$ $7900 \mathrm{~ms}, \mathrm{FOV}=864 \times 864 \mathrm{~mm}^{2}$, slice thickness $=1.4 \mathrm{~mm}$, in-plane resolution $=2 \mathrm{~mm}$, slice spacing $=1.4 \mathrm{~mm}$, slice duration $=123.2 \mathrm{~ms}$, number of slices $=35$, slice size: $104 \times 104$ pixels, diffusion sensitivity $\mathrm{b}=700 \mathrm{~s} / \mathrm{mm}^{2}$, and gradient directions $=192,64$ or 12 . In each direction, MRI scans were performed 6-10 times for noise reduction. For each heart, two different direction sampling strategies were employed to obtain two groups of gradient directions, one for $n$ directions and the other one for $2 n$ directions. Then their corresponding DW images were selected to train and test the network. In the following experiments, $n=6$ and 24 and one human heart will be reported here.

\section{B. Experimental initialization}

1) Gradient directions sampling: A necessary property for an optimal sampling scheme in dMRI scan is that the directions should be spherically uniformly distributed with no directional preference. In our method, given a set of directions, we generated a number of subsets of directions by using an effective direction Q-space sampling algorithm [22]. Each subset consists of $2 n$ directions. Then the sampling algorithm was used to select $n$ directions from each subset to form a pair of direction groups conditioning $n$ and $2 n$ directions, respectively. As a result, both the $n$ and $2 n$ gradient directions are distributed uniformly on a sphere.

2) Directional rotation: Since our network can just output DW image sequence without any directional information in q-space, it is necessary to determine a uniform input and output CNN training model, and thus results can be used in reconstruction of DTI without confusion of various directions for the generated DW images. Previous studies have shown that several independent sets of directions uniformly spread on the sphere can be regarded as rigid rotations of the others [23]. In our method, we first determine a fixed group of $2 n$ directions as the reference for the directions of input and output DW images. For each set with different directions, we find the optimal composition of rigid rotations with respect to the reference using a point matching algorithm. Then, the most uniform coverage possible is achieved by projecting the rotated sets of points onto the same reference sphere. Finally, the rotated DW images are taken as training and testing data. 


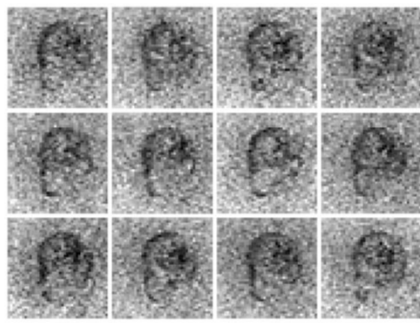

Generated images

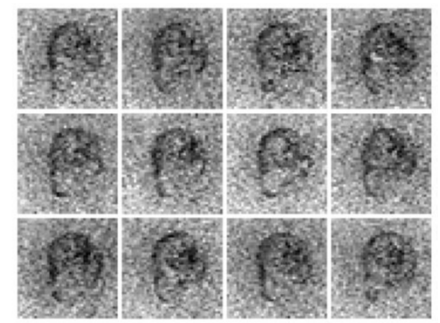

Ground-truth
Fig. 3. An example of generated DW images using our method. Here the 12 DW images were generated from 6 given directions for 12 gradient directions.

TABLE I

COMPARISON BETWEEN GENERATED AND GROUND TRUTH TENSOR METRICS.

\begin{tabular}{c|c|c|c|c}
\hline \hline & MSE & RMSE & PNSR & SSIM \\
\hline MD & 0.0002 & 0.0141 & 36.1151 & 0.9710 \\
AD & 0.0026 & 0.051 & 25.8167 & 0.8787 \\
FA & 0.0038 & 0.0616 & 24.2153 & 0.7633 \\
\hline \hline
\end{tabular}

\section{Results}

Fig. 3 shows 12 DW images generated from 6 acquired DW images by our method. The network was trained and tested by 600 and 400 samples, respectively. In the generated images, both the structural and detail information are preserved. This indicates that the generative model has well captured the underlying distribution of the input data, and thus generated realistic images. Furthermore, we also computed DT field and mapped it with three commonly used metrics computed from the three eigenvalues of the tensors: mean diffusivity (MD), axial diffusion (AD), and fractional anisotropy (FA) [24]. In Table I we quantitatively compared the difference between the $\mathrm{MD}, \mathrm{AD}, \mathrm{FA}$ from the generated DT and ground-truth. The results were tested in terms of mean squared error (MSE), root Mean squared error (RMSE), peak signal to noise ratio (PNSR), and structural similarity index (SSIM) [25]. We found that the $\mathrm{MD}$ and $\mathrm{AD}$ are more similar to their corresponding ground-truth compared with FA. This may be because FA are more sensitive to anisotropic diffusion within a voxel. In other words, our method can rather accurately simulate the signals from isotropic area, but failed to represent strongly anisotropic signals.

Table II gives a quantitative comparison of diffusion properties and fiber orientations for original DW images in 24 directions. The network was trained and tested by 1000 and 500 samples, respectively. The final resulting DW images consists of the original 24 DW images and new 24 DW images (in 24 directions) generated by the proposed method. The images in 192 direction were used as ground-truth for comparison. The values in the first three columns are the RMSE differences between the estimated DW images and ground-truth in terms of MD $\mathrm{AD}$, and $\mathrm{FA}$, respectively. The values in the last column are the angle differences between the underlying critical fiber orientations of the estimated images
TABLE II

RMSE OF DIFFUSION PROPERTIES AND FIBER ORIENTATIONS FOR ORIGINAL DW IMAGES IN 24 DIRECTIONS (TOP ROW) AND OUR SIMULATED DW IMAGES IN 48 DIRECTIONS (BOTTOM ROW).

\begin{tabular}{c|c|c|c|c}
\hline \hline & MD & AD & FA & difference (deg) \\
\hline Original & $0.206 \times 10^{-} 4$ & $0.569 \times 10^{-} 3$ & 0.0394 & 6.046 \\
Obtained & $0.142 \times 10^{-} 4$ & $0.381 \times 10^{-} 3$ & 0.0265 & 4.581 \\
\hline \hline
\end{tabular}

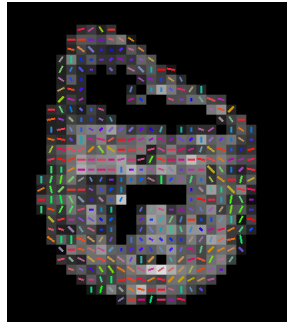

(a)

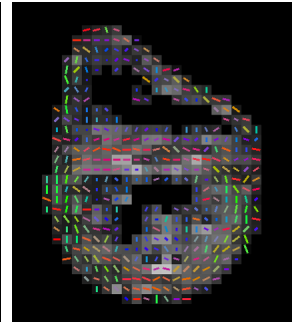

(b)

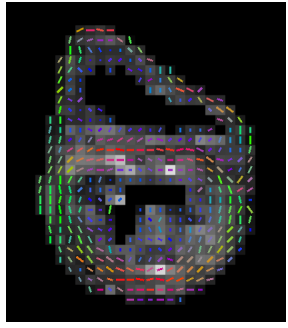

(c)
Fig. 4. An comparison of main fiber orientations. (a), (b) and (c) are the fiber orientations estimated from original DW images in 24 directions, our obtained DW images in 48 directions, and DW images in 192 directions as ground-truth, respectively.

and ground truth. An example of qualitative results in terms of main fiber orientations on one human heart is given in Fig. 4 with FA image as backgrounds. It can be seen in that the main fiber orientation from the our resulted DW images are much closer to the ground-truth, compared with the fiber orientation from original images. Note that the fiber orientations from the original DW images are very noisy, while the fiber orientations from the resulted DW images are locally well-aligned and regularized.

\section{CONCLUSION}

We have presented a deep leaning-based translation method for super-angular resolution in cardiac dMRI data. DW images in additional gradient directions are estimated from given DW image sequence acquired in a limited number of gradient directions. The correlation between multiple DW images acquired in different gradient directions are exploited by introducing a supervised manifold regularized term which takes advantage of the information of gradient directions in 3D q-space. Experiments on cardiac dMRI data showed that our method is capable of producing highly realistic dMRI data and improving the accuracy of DTI measurements.

\section{ACKNOWLEDGMENTS}

This work was funded in part by the Program PHC-Cai Yuanpei 2018 ( $\mathrm{N}^{\circ}$ 41400TC), and the CNRS International Research Project METISLAB.

\section{DECLARATIONS OF INTEREST: NONE.}

\section{REFERENCES}

[1] J.-M. Peyrat, M. Sermesant, X. Pennec, H. Delingette, C. Xu, E. R. McVeigh, and N. Ayache, "A computational framework for the statistical analysis of cardiac diffusion tensors: application to a small database of 
canine hearts," IEEE Transactions on Medical Imaging, vol. 26, no. 11, pp. 1500-1514, 2007.

[2] P. J. Basser, J. Mattiello, and D. LeBihan, "Mr diffusion tensor spectroscopy and imaging," Biophysical journal, vol. 66, no. 1, pp. 259-267, 1994.

[3] D. S. Tuch, T. G. Reese, M. R. Wiegell, N. Makris, J. W. Belliveau, and V. J. Wedeen, "High angular resolution diffusion imaging reveals intravoxel white matter fiber heterogeneity," Magnetic Resonance in Medicine: An Official Journal of the International Society for Magnetic Resonance in Medicine, vol. 48, no. 4, pp. 577-582, 2002.

[4] D. S. Tuch, "Q-ball imaging," Magnetic Resonance in Medicine: An Official Journal of the International Society for Magnetic Resonance in Medicine, vol. 52, no. 6, pp. 1358-1372, 2004.

[5] M. Descoteaux, E. Angelino, S. Fitzgibbons, and R. Deriche, "Regularized, fast, and robust analytical q-ball imaging," Magnetic Resonance in Medicine: An Official Journal of the International Society for Magnetic Resonance in Medicine, vol. 58, no. 3, pp. 497-510, 2007.

[6] R. G. Baraniuk, "Compressive sensing [lecture notes]," IEEE signal processing magazine, vol. 24, no. 4, pp. 118-121, 2007.

[7] J. Cheng, D. Shen, P. J. Basser, and P.-T. Yap, "Joint 6d kq space compressed sensing for accelerated high angular resolution diffusion mri," in International Conference on Information Processing in Medical Imaging. Springer, 2015, pp. 782-793.

[8] M. Mani, M. Jacob, A. Guidon, V. Magnotta, and J. Zhong, "Acceleration of high angular and spatial resolution diffusion imaging using compressed sensing with multichannel spiral data," Magnetic resonance in medicine, vol. 73, no. 1, pp. 126-138, 2015.

[9] A. Jog, A. Carass, and J. L. Prince, "Improving magnetic resonance resolution with supervised learning," in 2014 IEEE 11th International Symposium on Biomedical Imaging (ISBI). IEEE, 2014, pp. 987-990.

[10] T. Huynh, Y. Gao, J. Kang, L. Wang, P. Zhang, J. Lian, and D. Shen, "Estimating ct image from mri data using structured random forest and auto-context model," IEEE transactions on medical imaging, vol. 35, no. 1 , pp. 174-183, 2015.

[11] C. Dong, C. C. Loy, K. He, and X. Tang, "Image super-resolution using deep convolutional networks," IEEE transactions on pattern analysis and machine intelligence, vol. 38, no. 2, pp. 295-307, 2015.

[12] J. Kim, J. Kwon Lee, and K. Mu Lee, "Deeply-recursive convolutional network for image super-resolution," in Proceedings of the IEEE conference on computer vision and pattern recognition, 2016, pp. 1637-1645.

[13] I. Goodfellow, J. Pouget-Abadie, M. Mirza, B. Xu, D. Warde-Farley, S. Ozair, A. Courville, and Y. Bengio, "Generative adversarial nets," in Advances in neural information processing systems, 2014, pp. 2672 2680.

[14] A. Radford, L. Metz, and S. Chintala, "Unsupervised representation learning with deep convolutional generative adversarial networks," arXiv preprint arXiv:1511.06434, 2015.

[15] M. Mirza and S. Osindero, "Conditional generative adversarial nets," arXiv preprint arXiv:1411.1784, 2014.

[16] S. Ioffe and C. Szegedy, "Batch normalization: Accelerating deep network training by reducing internal covariate shift," arXiv preprint arXiv:1502.03167, 2015.

[17] O. Ronneberger, P. Fischer, and T. Brox, "U-net: Convolutional networks for biomedical image segmentation," in International Conference on Medical image computing and computer-assisted intervention. Springer, 2015, pp. 234-241.

[18] P. Isola, J.-Y. Zhu, T. Zhou, and A. A. Efros, "Image-to-image translation with conditional adversarial networks," in Proceedings of the IEEE conference on computer vision and pattern recognition, 2017, pp. 11251134.

[19] D. Pathak, P. Krahenbuhl, J. Donahue, T. Darrell, and A. A. Efros, "Context encoders: Feature learning by inpainting," in Proceedings of the IEEE conference on computer vision and pattern recognition, 2016, pp. 2536-2544.

[20] Q. Li, B. Kailkhura, R. Anirudh, Y. Zhou, Y. Liang, and P. Varshney, "Mr-gan: Manifold regularized generative adversarial networks," arXiv preprint arXiv:1811.10427, 2018.

[21] X. He and P. Niyogi, "Locality preserving projections," in Advances in neural information processing systems, 2004, pp. 153-160.

[22] J. Cheng, D. Shen, P.-T. Yap, and P. J. Basser, "Single-and multipleshell uniform sampling schemes for diffusion mri using spherical codes," IEEE transactions on medical imaging, vol. 37, no. 1, pp. 185-199, 2018.
[23] E. Caruyer, C. Lenglet, G. Sapiro, and R. Deriche, "Design of multishell sampling schemes with uniform coverage in diffusion mri," Magnetic resonance in medicine, vol. 69, no. 6, pp. 1534-1540, 2013.

[24] R. Kumar, R. K. Gupta, M. Husain, C. Chaudhry, A. Srivastava, S. Saksena, and R. K. Rathore, "Comparative evaluation of corpus callosum dti metrics in acute mild and moderate traumatic brain injury: its correlation with neuropsychometric tests," Brain injury, vol. 23, no. 7-8, pp. 675-685, 2009.

[25] U. Sara, M. Akter, and M. S. Uddin, "Image quality assessment through fsim, ssim, mse and psnr-a comparative study," Journal of Computer and Communications, vol. 7, no. 3, pp. 8-18, 2019. 